\title{
Article \\ A Comprehensive, Targeted NGS Approach to Assessing Molecular Diagnosis of Lysosomal Storage Diseases
}

\author{
Valentina La Cognata and Sebastiano Cavallaro *(D) \\ Institute for Biomedical Research and Innovation (IRIB), National Research Council (CNR), 95126 Catania, Italy; \\ valentina.lacognata@cnr.it \\ * Correspondence: sebastiano.cavallaro@cnr.it; Tel.: +39-0957338111
}

Citation: La Cognata, V.; Cavallaro, S. A Comprehensive, Targeted NGS Approach to Assessing Molecular Diagnosis of Lysosomal Storage Diseases. Genes 2021, 12, 1750. https://doi.org/10.3390/ genes 12111750

Academic Editor: Hirokazu Takahashi

Received: 6 September 2021 Accepted: 27 October 2021 Published: 30 October 2021

Publisher's Note: MDPI stays neutral with regard to jurisdictional claims in published maps and institutional affiliations.

Copyright: (c) 2021 by the authors. Licensee MDPI, Basel, Switzerland. This article is an open access article distributed under the terms and conditions of the Creative Commons Attribution (CC BY) license (https:// creativecommons.org/licenses/by/ $4.0 /)$.

\begin{abstract}
With over 60 different disorders and a combined incidence occurring in 1:5000-7000 live births, lysosomal storage diseases (LSDs) represent a major public health problem and constitute an enormous burden for affected individuals and their families. Several reasons make the diagnosis of LSDs an arduous task for clinicians, including the phenotype and penetrance variability, the shared signs and symptoms, and the uncertainties related to biochemical enzymatic assay results. Developing a powerful diagnostic tool based on next generation sequencing (NGS) technology may help reduce the delayed diagnostic process for these families, leading to better outcomes for current therapies and providing the basis for more appropriate genetic counseling. Herein, we employed a targeted NGS-based panel to scan the coding regions of 65 LSD-causative genes. A reference group sample $(n=26)$ with previously known genetic mutations was used to test and validate the entire workflow. Our approach demonstrated elevated analytical accuracy, sensitivity, and specificity. We believe the adoption of comprehensive targeted sequencing strategies into a routine diagnostic route may accelerate both the identification and management of LSDs with overlapping clinical profiles, producing a significant reduction in delayed diagnostic response with beneficial results in the treatment outcome.
\end{abstract}

Keywords: lysosomal storage disease (LSDs); diagnosis; targeted next generation sequencing (tNGS)

\section{Introduction}

Lysosomal storage disorders (LSDs) are rare inherited diseases characterized by the accumulation of specific undegraded metabolites inside the lysosomes [1-3]. This overstorage is commonly caused by a deficiency or absent activity of lysosomal hydrolases or, in a few cases, by the deficit of further non-enzymatic lysosomal proteins (such as integral membrane proteins) [3]. With a combined incidence of 1 in 1500 to 7000 live births, this group of monogenic inborn errors of metabolism encompasses $\sim 70$ different entities, including sphingolipidoses, mucopolysaccharidoses, glycoproteinoses, lipid storage diseases, lipofuscinosis, lysosomal integral membrane proteins diseases, and post-translational modifications dysfunctions [4,5]. Clinical signs and symptoms may occur from the prenatal period to adulthood and may develop progressively over time, leading to a wide spectrum of disease phenotypes from mild to extremely severe forms that involve neuropathological effects, psychomotor development delay, cognitive decline, musculoskeletal abnormalities, dysmorphia, organomegaly, and seizures [6]. Both the considerable clinical variability within each disease phenotype and the overlapping symptomatology among single LSDs hamper the path for a precise diagnosis, which often involves a delay in treatment and severe consequences on patients' quality of life and their families [4].

Current diagnostic workflows include an accurate evaluation of both medical history and clinical presentations, which lead to the formulation of suspicion of one or more LSDs, followed by biochemical analysis to quantify either the accumulated storage product or the enzymatic activity in leukocytes, fibroblasts, urine, or rehydrated dried blood spots (DBS) 
for newborns [7,8]. Finally, if deficient enzyme activity is detected, second-tier confirmatory biomarker tests or Sanger sequencing are performed for the suspected gene. Although this diagnostic route represents the current gold standard, it presents several limitations. First, it requires deep clinical expertise to discriminate phenotypic overlapping manifestations and, thus, to reduce the number of biochemical tests used for each LSD-suspected patient. Second, the execution of multiple biochemical enzymatic assays may be expensive, timeconsuming, and subject to high variability, and enzymatic tests may not be available for all diseases. Therefore, reaching a definitive molecular diagnosis for LSDs with traditional techniques is still challenging, can take several years, or may be unsuccessful.

In the past decade, the emergence of next generation sequencing (NGS) technologies has proven to be an effective alternative to conventional techniques, in both research and clinical settings, allowing for the simultaneous interrogation of several genes in one single reaction and reducing, considerably, the time and costs for Sanger sequencing of a single gene $[9,10]$. The introduction of ad hoc designed genetic tests (targeted NGS panels) into diagnostic workflows offers the opportunity for easier identification of LSDs, timely diagnosis, and optimized clinical management, reducing the psychological burden and providing appropriate genetic counseling to parents [4].

In this study, we aimed to design and evaluate both the diagnostic utility of a semiautomated and comprehensive sequencing assay based on a targeted NGS (tNGS) panel (hereafter referred to as LSDs_panel) developed to detect pathogenic variants in 65 LSDrelated genes. We describe the panel performance, strengths, and limitations and propose it as a useful second-tier diagnostic test for specialists in everyday clinical management who might suspect an LSD, given its ability to provide accurate and timely information.

\section{Materials and Methods}

\subsection{Sample Collection and Dosage}

A reference group of DNA samples isolated from clinically diagnosed donor subjects ( $n=26)$ were obtained from the NIGMS Human Genetic Cell Repository at the Coriell Institute for Medical Research (https:/ / www.coriell.org/, accessed on 26 October 2021). The purchased samples were chosen for known variants localized in targeted genes and selected in order to ensure an adequate representation for most LSDs. Quantification of the genomic DNA was assessed by measuring the genomic copies of the human RNase P gene using the TaqMan ${ }^{\circledR}$ RNase P Detection Reagents Kit (Thermo Fisher Scientific, Waltham, MA, USA) and the Aria Dx Real-Time PCR System (Agilent Technologies, Santa Clara, CA, USA).

\subsection{Panel Design and Library Preparation}

For the selection of genes $(n=65)$ included in the panel, we relied on updated literature data [2] and a previous gene-set used for targeted strategies (Table 1). An on-demand panel (IAD199901) and a compatible made-to-order spike-in panel (IAD199905 including TPP1 and BLOC1S3 genes) were designed using the Ion AmpliSeq Designer software (https: / / ampliseq.com, accessed on 1 May 2020, Thermo Fisher Scientific, Waltham, MA, USA). The advantage of using Ion AmpliSeq on-demand panel customization is that primer pairs are pre-tested and optimized for high performance, whereas spike-ins are high concentrated made-to-order panels used to extend panels for genes not available on-demand.

The complete panel design (called LSDs_panel) covers $237.782 \mathrm{~kb}$ and includes 1241 amplicons with a size range of 125-275 bp distributed across two primer pools (625 primer pool 1 and 616 primer pool 2). The in silico coverage consisted of $99 \%$ for the on-demand panel and $99.18 \%$ for the spike-ins. The complete design of the LSDs_panel is available in Supplementary Table S1.

Library preparation was carried out using the Ion AmpliSeq ${ }^{\mathrm{TM}}$ Kit for Chef DL8 (DNA to Library, 8 samples/run) used for automated library preparation of the Ion AmpliSeq ${ }^{\mathrm{TM}}$ libraries on the Ion Chef ${ }^{\mathrm{TM}}$ System (Thermo Fisher Scientific, Waltham, MA, USA). According to the recommended number of amplification cycles in the standard protocol, the 
amplification conditions were set out to 16 cycles and four minutes of annealing/extension time. The library quality and molarity were assessed using the Ion Library TaqMan ${ }^{\circledR}$ Quantitation Kit (Thermo Fisher Scientific, Waltham, MA, USA) on the Aria Dx Real-Time PCR System (Agilent Technologies, Santa Clara, CA, USA). Serial dilutions of the E. coli DH10B Control Library (Thermo Fisher Scientific, Waltham, MA, USA) were prepared and run in triplicate to generate a standard curve. The molar concentration of libraries was determined using the Delta $\mathrm{R}$-baseline-corrected raw fluorescence calculated with Aria DX Real-Time PCR Software (Agilent Technologies, Santa Clara, CA, USA). Barcoded libraries (up to 4-Chef runs corresponding to 32 libraries) were super-pooled in equimolar concentration using the strategies suggested for combining libraries prepared with different panels for equal coverage in order to obtain a final molarity of $40 \mathrm{pM}$ each.

\subsection{Chip Loading and Sequencing}

Loading of the Ion 510 and the 540 Chips was carried out using the Ion 510, 520, 530, and 540 Kit-Chef (Thermo Fisher Scientific, Waltham, MA, USA) following manufacturer instructions. High throughput sequencing runs were carried out on the Ion Gene Studio S5 system (Thermo Fisher Scientific, Waltham, MA, USA). A run planned in the S5 Torrent Suite (v. 5.12.2) had the following parameters: analysis parameters, default; reference library, hg19; target regions, LSDs_panel; read length, 200 bp; flows, 550; and base calibration mode, default. The plugins used were Coverage Analysis, Ion Reporter Uploader, and Variant Caller (default settings).

\subsection{Variant Calling and Prioritization}

Read mapping was performed automatically in Torrent Suite (v. 5.12.2, Thermo Fisher Scientific, Waltham, MA, USA) by using the variant Caller plugin (v5.12.0.4) with default settings (germline_low_stringency). The called variants were automatically uploaded on Ion Reporter (Thermo Fisher Scientific, Waltham, MA, USA). The Copy Number Variation (CNV) performance was not assessed. The pipeline analysis for variant filtering was based on multiple adjusted steps including coverage min $30 \times$, Homopolymer length $\leq 3$, $p$-value $<0.001$, ClinVar $\neq$ benign or likely benign, MAF $<0.001$ or n.a., frequency $30-60 \%$ for heterozygous variants and $>70 \%$ for homozygous variants, intronic variants included if the distance from exon is $<10 \mathrm{bp}$, SIFT score $<0.05 /$ PolyPhen score $>0.85$ or n.a., and variants effect $\neq$ synonymous unless they are pathogenic/likely pathogenic or with conflicting interpretation of pathogenicity. A comparison of the Torrent Variant Caller (TVC) prioritized variants with their respective genetic information from Coriell biobank was performed post-analysis. True-positive (TP), true-negatives (TN), false-positive (FP), and false-negative (FN) variant calls were defined by considering available data from the single causative gene in the Coriell repository. True positives (TPs) were defined as variants both detected by our filtering pipeline as well as expected from the Coriell collected data. True negatives (TNs) were considered additional variants detected in the causative gene but excluded by our prioritization pipeline and not reported in the repository data. False positives (FPs) were considered variants detected by our pipeline but not expected from the data. False negatives (FNs) were considered variants expected from the Coriell data but missed by our pipeline. Accuracy was calculated as follows: $(\mathrm{TP}+\mathrm{TN}) /(\mathrm{TP}+\mathrm{FP}+\mathrm{TN}+\mathrm{FN})$; sensitivity was calculated as follows: TP/(TP $+\mathrm{FN})$; and specificity was calculated as follows: $\mathrm{TN} /(\mathrm{TN}+\mathrm{FP}$ ). The Matthews correlation coefficient (MCC) (which measures the correlation between the predicted and observed binary classification of a sample) was calculated as follows: $\mathrm{MCC}=[(\mathrm{TP} \times \mathrm{TN})-(\mathrm{FP} \times \mathrm{FN})] / \sqrt{ }[(\mathrm{TP}+\mathrm{FP})(\mathrm{TP}+\mathrm{FN})(\mathrm{TN}+\mathrm{FP})(\mathrm{TN}+\mathrm{FN})]$.

\section{Results}

\subsection{Panel Design and Performance}

The LSDs_panel was designed to target the entire coding regions of 65 LSD-related genes (Table 1), which were previously reported to be a direct cause of an LSD when mutated in both alleles, in order to use it for diagnostic testing in patients with a high a priori 
probability of LSD based on the clinical phenotype. The LSDs_panel included 1241 amplicons (with a length of $125-275 \mathrm{bp})$ distributed between two primer pools $(625+616$ primer pairs) and covering a size of $237.782 \mathrm{~kb}$, with an in silico coverage of $99 \%$ (the complete design of LSDs_panel is available in Supplementary Table S1). No additional intronic regions were targeted to maximize the coverage of exonic regions and to facilitate rapid and unambiguous interpretation in the context of diagnosis.

Table 1. LSD-related genes included in the panel and their associated disorders.

\begin{tabular}{|c|c|c|c|}
\hline Gene & Cytogenetic Location & Pathology & $\begin{array}{l}\text { Phenotype } \\
\text { OMIM No. }\end{array}$ \\
\hline$A G A$ & $4 q 34.3$ & Aspartylglucosaminuria & 208400 \\
\hline$A P 3 B 1$ & $5 q 14.1$ & Hermansky-Pudlak disease type 2 & 608233 \\
\hline$A R S A$ & 22q13.33 & Metachromatic leukodystrophy & 250100 \\
\hline$A R S B$ & $5 q 14.1$ & MPS VI, also known as Maroteaux-Lamy syndrome & 253200 \\
\hline ASAH1 & $8 \mathrm{p} 22$ & Farber lipogranulomatosis & 228000 \\
\hline ATP13A2 & $1 \mathrm{p} 36.13$ & CLN12b: Kufor-Rakeb syndrome or PARK9 & 606693 \\
\hline BLOC1S6 & $15 \mathrm{q} 21.1$ & Hermansky-Pudlak disease type 9 & 614171 \\
\hline BLOCS13 & $19 q 13.32$ & Hermansky-Pudlak disease type 8 & 614077 \\
\hline CLN3 & $16 \mathrm{p} 12.1$ & CLN3: Batten-Spielmeyer-Sjogren disease & 204200 \\
\hline CLN5 & $13 q 22.3$ & CLN5: Finnish variant late infantile & 256731 \\
\hline CLN6 & $15 q 23$ & CLN6: Lake-Cavanagh or Indian variant & 601780 \\
\hline CLN8 & $8 \mathrm{p} 23.3$ & CLN8: northern epilepsy, epilepsy mental retardation & $\begin{array}{l}600143 \\
610003\end{array}$ \\
\hline CTNS & $17 \mathrm{p} 13.2$ & Cystinosis & 219800 \\
\hline CTSA & $20 \mathrm{q} 13.12$ & Galactosialidosis & 256540 \\
\hline CTSD & $11 \mathrm{p} 15.5$ & CLN10 & 610127 \\
\hline CTSF & $11 \mathrm{q} 13.2$ & CLN13 & 615362 \\
\hline DNAJC5 & $20 q 13.33$ & CLN4: Parry disease and Kufs type A and B & 162350 \\
\hline DTNBP1 & 6p22.3 & Hermansky-Pudlak disease type 7 & 614076 \\
\hline FUCA1 & $1 \mathrm{p} 36.11$ & Fucosidosis & 230000 \\
\hline$G A A$ & $17 q 25.3$ & Pompe disease & 232300 \\
\hline GALC & $14 \mathrm{q} 31.3$ & Globoid cell leukodystrophy, Krabbe disease & 245200 \\
\hline GALNS & $16 \mathrm{q} 24.3$ & MPS IVA, also known as Morquio syndrome A & 253000 \\
\hline$G B A$ & $1 \mathrm{q} 22$ & Gaucher disease & 230800 \\
\hline GLA & Xq22.1 & Fabry disease & 301500 \\
\hline GLB1 & 3 p22.3 & GM1 gangliosidosis; MPS IVB, also known as Morquio syndrome B & 253010 \\
\hline$G M 2 A$ & $5 q 33.1$ & GM2 gangliosidosis, GM2 activator deficiency & 272750 \\
\hline GNPTAB & $12 q 23.2$ & $\begin{array}{l}\text { Mucolipidosis II } \alpha / \beta \text {, I-cell disease; mucolipidosis III } \alpha / \beta \text {, } \\
\text { pseudo-Hurler polydystrophy }\end{array}$ & $\begin{array}{l}252500 \\
252600\end{array}$ \\
\hline GNPTG & $16 \mathrm{p} 13.3$ & Mucolipidosis III $\gamma$, variant pseudo-Hurler polydystrophy & 252605 \\
\hline GNS & $12 \mathrm{q} 14.3$ & MPS IIID, also known as Sanfilippo syndrome D & 252940 \\
\hline GRN & $17 q 21.31$ & CLN11 & 614706 \\
\hline GUSB & $7 q 11.21$ & MPS VII, also known as Sly disease & 253220 \\
\hline HEXA & $15 q 23$ & GM2 gangliosidosis, Tay-Sachs disease & 272800 \\
\hline HEXB & $5 q 13.3$ & GM2 gangliosidosis, Sandhoff diseaseb & 268800 \\
\hline HGSNAT & 8p11.2-p11.1 & MPS IIIC, also known as Sanfilippo syndrome C & 252930 \\
\hline HPS1 & $10 \mathrm{q} 24.2$ & Hermansky-Pudlak disease type 1 & 203300 \\
\hline HPS3 & $3 q 24$ & Hermansky-Pudlak disease type 3 & 614072 \\
\hline HPS4 & $22 q 12.1$ & Hermansky-Pudlak disease type 4 & 614073 \\
\hline HPS5 & 11p15.1 & Hermansky-Pudlak disease type 5 & 614074 \\
\hline HPS6 & $10 \mathrm{q} 24.32$ & Hermansky-Pudlak disease type 6 & 614075 \\
\hline HYAL1 & $3 p 21.31$ & MPS IX & 601492 \\
\hline IDS & $\mathrm{Xq} 28$ & MPS II, also known as Hunter syndrome & $\begin{array}{l}309900 \\
607014\end{array}$ \\
\hline IDUA & $4 \mathrm{p} 16.3$ & MPS I: Hurler syndrome & $\begin{array}{l}607015 \\
607016\end{array}$ \\
\hline KCTD7 & $7 q 11.21$ & CLN14 & 611726 \\
\hline
\end{tabular}


Table 1. Cont.

\begin{tabular}{|c|c|c|c|}
\hline Gene & Cytogenetic Location & Pathology & $\begin{array}{l}\text { Phenotype } \\
\text { OMIM No. }\end{array}$ \\
\hline LAMP2 & $\mathrm{Xq} 24$ & Danon disease & 300257 \\
\hline LIPA & $10 q 23.31$ & $\begin{array}{c}\text { Acid lipase deficiency: Wolman disease and cholesterol ester storage } \\
\text { disease }\end{array}$ & 278000 \\
\hline LYST & $1 q 42.3$ & Chédiak-Higashi disease & 214500 \\
\hline MAN2B1 & $19 \mathrm{p} 13.13$ & $\alpha$-Mannosidosis & 248500 \\
\hline$M A N B A$ & $4 q 24$ & $\beta$-Mannosidosis & 248510 \\
\hline MCOLN1 & $19 \mathrm{p} 13.2$ & Mucolipidosis IV & 252650 \\
\hline MFSD8 & $4 \mathrm{q} 28.2$ & CLN7: Turkish variant & 610951 \\
\hline MYO5A & $15 \mathrm{q} 21.2$ & Griscelli syndrome 1, also known as Elejalde syndrome & 214450 \\
\hline NAGA & $22 \mathrm{q} 13.2$ & $\begin{array}{l}\text { Schindler disease: type } \mathrm{Ib} \text {, also known as infantile-onset neuroaxonal } \\
\text { dystrophy, type IIb also known as Kanzaki disease, and type IIIb, } \\
\text { intermediate severity }\end{array}$ & $\begin{array}{l}609241 \\
609242\end{array}$ \\
\hline NAGLU & $17 \mathrm{q} 21.2$ & MPS IIIB, also known as Sanfilippo syndrome B & 252920 \\
\hline NEU1 & $6 \mathrm{p} 21.33$ & Sialidosis type I, Sialidosis type II & 256550 \\
\hline NPC1 & $18 \mathrm{q} 11.2$ & Niemann-Pick disease types C1 & 257220 \\
\hline NPC2 & $14 \mathrm{q} 24.3$ & Niemann-Pick disease types C1 and C2 & 607625 \\
\hline PPT1 & $1 \mathrm{p} 34.2$ & CLN1: Haltia-Santavuori disease and INCL & 256730 \\
\hline PSAP & $10 \mathrm{q} 22.1$ & Metachromatic leukodystrophy & 249900 \\
\hline$R A B 27 A$ & $15 q 21.3$ & Griscelli syndrome 2 & 607624 \\
\hline SCARB2 & $4 q 21.1$ & Action myoclonus-renal failure syndrome & 254900 \\
\hline SGSH & $17 \mathrm{q} 25.3$ & MPS IIIA, also known as Sanfilippo syndrome A & 252900 \\
\hline SLC17A5 & $6 q 13$ & Sialic acid storage disease & 269920 \\
\hline SMPD1 & $11 \mathrm{p} 15.4$ & Niemann-Pick disease types A and B & $\begin{array}{l}257200 \\
607616\end{array}$ \\
\hline SUMF1 & $3 \mathrm{p} 26.1$ & Multiple sulfatase deficiency & 272200 \\
\hline TPP1 & $11 \mathrm{p} 15.4$ & CLN2, also known as Jansky-Bielschowsky disease & 204500 \\
\hline
\end{tabular}

Before investigating the clinical utility of the gene panel, we sought to determine the analytical performance of our method in terms of depth of coverage across all targeted genes. Therefore, we used a reference group of DNA samples $(n=26$, Table 2$)$, isolated from clinically diagnosed donors from the NIGMS Human Genetic Cell Repository at the Coriell Institute for Medical Research and previously Sanger-sequenced for the LSDsuspected genes.

Table 2. Detected and missed pathogenic variants in reference samples from Coriell repository.

\begin{tabular}{|c|c|c|c|c|c|c|c|}
\hline $\begin{array}{l}\text { ID Coriell } \\
\text { Sample }\end{array}$ & Genes & Zigosity & Transcript & $\begin{array}{l}\text { Coding Amino Acid } \\
\text { Change }\end{array}$ & Variant Effect & dbSNP & ClinVar \\
\hline NA03392 & GNPTG & Hom & NM_032520.5 & $\begin{array}{c}\text { c.445delG } \\
\text { p.Ala149Profs Ter13 }\end{array}$ & frameshiftDeletion & rs1555451874 & $\mathbf{P}$ \\
\hline \multirow{2}{*}{ NA03461 } & \multirow{2}{*}{$H E X A$} & \multirow{2}{*}{ Het } & \multirow{2}{*}{ NM_000520.6 } & $\begin{array}{c}\text { c. } 1421+1 G>C \\
\text { p.? }\end{array}$ & unknown & rs147324677 & $\mathbf{P}$ \\
\hline & & & & $\begin{array}{c}\text { c.805G>A } \\
\text { p.Gly269Ser }\end{array}$ & missense & rs121907954 & P/LP \\
\hline NA05093 & GNS & Hom & NM_002076.4 & $\begin{array}{c}\text { c.1063C>T } \\
\text { p.Arg355Ter }\end{array}$ & nonsense & rs119461974 & $\mathbf{P}$ \\
\hline \multirow{3}{*}{ NA00654 } & GLB1 & Het & NM_000404.4 & $\begin{array}{l}\text { c. } 1032 \mathrm{~T}>\mathrm{C} \\
\text { p.Thr344= }\end{array}$ & synonymous & rs199927127 & CIP \\
\hline & \multirow{2}{*}{$M A N 2 B 1$} & \multirow{2}{*}{ Het } & \multirow{2}{*}{ NM_000528.4 } & $\begin{array}{c}\text { c. } 2248 \mathrm{C}>\mathrm{T} \\
\text { p.Arg750Trp }\end{array}$ & missense & rs80338680 & $\mathbf{P}$ \\
\hline & & & & $\begin{array}{c}\text { c.1915C>T } \\
\text { p.Gln639Ter }\end{array}$ & nonsense & rs121434332 & $\mathbf{P}$ \\
\hline
\end{tabular}


Table 2. Cont.

\begin{tabular}{|c|c|c|c|c|c|c|c|}
\hline $\begin{array}{l}\text { ID Coriell } \\
\text { Sample }\end{array}$ & Genes & Zigosity & Transcript & $\begin{array}{l}\text { Coding Amino Acid } \\
\text { Change }\end{array}$ & Variant Effect & dbSNP & ClinVar \\
\hline \multirow{2}{*}{ NA02528 } & AP3B1 & Het & NM_003664.5 & $\begin{array}{c}\text { c. } 1168-9 \mathrm{C}>\mathrm{T} \\
\text { p.? }\end{array}$ & unknown & rs367648410 & CIP \\
\hline & MCOLN1 & Hom & NM_020533.3 & $\begin{array}{c}\text { c. } 406-2 A>G \\
\text { p.? }\end{array}$ & unknown & rs104886461 & $\mathbf{P}$ \\
\hline \multirow{2}{*}{ NA01675 } & MFSD8 & Het & NM_152778.3 & $\begin{array}{c}\text { c.590G }>\text { A } \\
\text { p.Gly197Asp }\end{array}$ & missense & rs28544073 & CIP \\
\hline & $G M 2 A$ & Hom & NM_000405.5 & $\begin{array}{c}\text { c.412T>C } \\
\text { p.Cys138Arg }\end{array}$ & missense & rs137852797 & $\mathbf{P}$ \\
\hline \multirow{3}{*}{ NA02455 } & \multirow{2}{*}{ GLB1 } & \multirow{2}{*}{ Het } & \multirow{2}{*}{ NM_000404.4 } & $\begin{array}{c}\text { c.1445G >A } \\
\text { p.Arg482His }\end{array}$ & missense & rs72555391 & $\mathbf{P}$ \\
\hline & & & & $\begin{array}{c}\text { c.817_818delTGinsCT } \\
\text { p.Trp273Leu }\end{array}$ & missense & rs1559401428 & P/LP \\
\hline & CLN6 & Het & NM_017882.3 & $\begin{array}{c}\text { c. } 821 C>T \\
\text { p.Ala274Val }\end{array}$ & missense & rs202012876 & US \\
\hline \multirow{3}{*}{ NA02013 } & \multirow{2}{*}{ GNPTAB } & \multirow{2}{*}{ Het } & \multirow{2}{*}{ NM_024312.5 } & $\begin{array}{c}\text { c.3501_3502delTC } \\
\text { p.Leu1168GlnfsTer5 }\end{array}$ & frameshiftDeletion & rs34002892 & $\mathbf{P}$ \\
\hline & & & & $\begin{array}{c}\text { c.3233_3234insCCTA } \\
\text { p.Tyr1079LeufsTer3 }\end{array}$ & frameshiftInsertion & - & n.a. \\
\hline & GNPTG & Het & NM_032520.5 & $\begin{array}{l}\text { c. } 574 G>C \\
\text { p.Glu192Gln }\end{array}$ & missense & rs749314645 & US \\
\hline \multirow{4}{*}{ NA02552 } & GLB1 & Het & NM_000404.4 & $\begin{array}{c}\text { c. } 602 \mathrm{G}>\mathrm{A} \\
\text { p.Arg201His }\end{array}$ & missense & rs189115557 & $\mathrm{P}$ \\
\hline & HPS1 & Het & NM_000195.5 & $\begin{array}{c}\text { c. } 29 \mathrm{G}>\mathrm{T} \\
\text { p.Gly10Val }\end{array}$ & missense & rs759539605 & n.a. \\
\hline & \multirow{2}{*}{$N A G L U$} & \multirow{2}{*}{ Het } & \multirow{2}{*}{ NM_000263.4 } & $\begin{array}{c}\text { c.889C>T } \\
\text { p.Arg297Ter }\end{array}$ & nonsense & rs104894592 & P/LP \\
\hline & & & & $\begin{array}{c}\text { c.1928G >A } \\
\text { p.Arg643His }\end{array}$ & missense & rs104894593 & US \\
\hline NA17881 & HPS6 & Hom & NM_024747.6 & $\begin{array}{l}\text { c.1714_1717delCTGT } \\
\text { p.Leu572AlafsTer40 }\end{array}$ & frameshiftDeletion & rs281865113 & $\mathbf{P}$ \\
\hline \multirow{3}{*}{ NA17890 } & LYST & Het & NM_000081.4 & $\begin{array}{l}\text { c. } 149 \mathrm{G}>\mathrm{A} \\
\text { p.Arg50Gln }\end{array}$ & missense & rs368095341 & n.a. \\
\hline & \multirow{2}{*}{ AP3B1 } & \multirow{2}{*}{ Het } & \multirow{2}{*}{ NM_003664.5 } & $\begin{array}{c}\text { c.1975G >T } \\
\text { p.Glu659Ter }\end{array}$ & nonsense & rs121908907 & $\mathbf{P}$ \\
\hline & & & & $\begin{array}{c}\text { c.1525C>T } \\
\text { p.Arg509Ter }\end{array}$ & nonsense & rs121908906 & $\mathbf{P}$ \\
\hline NA17721 & SLC17A5 & Hom & NM_012434.5 & $\begin{array}{c}\text { c.115C>T } \\
\text { p.Arg39Cys }\end{array}$ & missense & rs80338794 & $\mathbf{P}$ \\
\hline \multirow{2}{*}{ NA16081 } & \multirow{2}{*}{ PPT1 } & \multirow{2}{*}{ Het } & \multirow{2}{*}{ NM_000310.4 } & $\begin{array}{c}\text { c.451C>T } \\
\text { p.Arg151Ter }\end{array}$ & nonsense & rs137852700 & P/LP \\
\hline & & & & $\begin{array}{c}\text { c.236A>G } \\
\text { p.Asp79Gly }\end{array}$ & missense & rs137852697 & $\mathbf{P}$ \\
\hline \multirow{3}{*}{ NA13204 } & DTNBP1 & Het & NM_032122.5 & $\begin{array}{l}\text { c.489_490insT } \\
\text { p.Lys164Ter }\end{array}$ & nonsense & - & n.a. \\
\hline & \multirow{2}{*}{$H E X A$} & \multirow{2}{*}{ Het } & \multirow{2}{*}{ NM_000520.6 } & $\begin{array}{l}\text { c.1277_1278insTATC } \\
\text { p.Tyr427IlefsTer5 }\end{array}$ & frameshiftInsertion & rs387906309 & $\mathbf{P}$ \\
\hline & & & & $\begin{array}{c}\text { c.805G }>A \\
\text { p.Gly269Ser }\end{array}$ & missense & rs121907954 & P/LP \\
\hline \multirow{3}{*}{ NA18455 } & $M A N B A$ & Het & NM_005908.4 & $\begin{array}{l}\text { c.1442A>C } \\
\text { p.Tyr481Ser }\end{array}$ & missense & rs764041854 & n.a. \\
\hline & \multirow{2}{*}{ NPC2 } & \multirow{2}{*}{ Het } & \multirow{2}{*}{ NM_006432.5 } & $\begin{array}{l}\text { c.140G >T } \\
\text { p.Cys47Phe }\end{array}$ & missense & rs1555345993 & US \\
\hline & & & & $\begin{array}{c}\text { c.58G }>\text { T } \\
\text { p.Glu20Ter }\end{array}$ & nonsense & rs80358260 & $\mathbf{P}$ \\
\hline
\end{tabular}


Table 2. Cont.

\begin{tabular}{|c|c|c|c|c|c|c|c|}
\hline $\begin{array}{l}\text { ID Coriell } \\
\text { Sample }\end{array}$ & Genes & Zigosity & Transcript & $\begin{array}{l}\text { Coding Amino Acid } \\
\text { Change }\end{array}$ & Variant Effect & dbSNP & ClinVar \\
\hline \multirow{3}{*}{ NA20387 } & \multirow{2}{*}{ TPP1 } & \multirow{2}{*}{ Het } & \multirow{2}{*}{ NM_000391.4 } & $\begin{array}{c}\text { c.622C>T } \\
\text { p.Arg208Ter }\end{array}$ & nonsense & rs119455955 & $\mathbf{P}$ \\
\hline & & & & $\begin{array}{c}\text { c.509-1G }>C \\
\text { p.? }\end{array}$ & unknown & rs56144125 & $\mathbf{P}$ \\
\hline & GALNS & Het & NM_000512.5 & $\begin{array}{l}\text { c. } 858 G>A \\
\text { p.Thr286= }\end{array}$ & synonymous & rs140299014 & CIP \\
\hline \multirow{3}{*}{ NA20019 } & \multirow{2}{*}{$A S A H 1$} & \multirow{2}{*}{ Het } & \multirow{2}{*}{ NM_004315.6 } & $\begin{array}{c}\text { c.1039G>A } \\
\text { p.Asp347Asn }\end{array}$ & missense & rs1354060089 & US \\
\hline & & & & $\begin{array}{c}\text { c.460G }>\mathrm{T} \\
\text { p.Glu154Ter }\end{array}$ & nonsense & rs1588982399 & $\mathbf{L P}$ \\
\hline & GNPTAB & Het & NM_024312.5 & $\begin{array}{c}\text { c.2708_2710delTTC } \\
\text { p.Leu904del }\end{array}$ & nonframeshiftDeletion & rs774128798 & US \\
\hline \multirow{2}{*}{ NA10866 } & IDUA & Het & NM_000203.5 & $\begin{array}{c}\text { c.785A }>\text { G } \\
\text { p.His262Arg }\end{array}$ & missense & rs1031451164 & n.a. \\
\hline & IDS & Hom & NM_000202.8 & $\begin{array}{c}\text { c.1403G>C } \\
\text { p.Arg468Pro }\end{array}$ & missense & rs113993946 & $\mathbf{P}$ \\
\hline \multirow{3}{*}{ NA12928 } & HPS1 & Hom & NM_000195.5 & $\begin{array}{l}\text { c.1484_1485insCCCCC- } \\
\text { AGCAGGGGAGG } \\
\text { p.His497GlnfsTer90 }\end{array}$ & frameshiftInsertion & - & n.a. \\
\hline & HPS6 & Het & NM_024747.6 & $\begin{array}{l}\text { c. } 2250 G>A \\
\text { p.Ser } 750=\end{array}$ & synonymous & rs139161525 & CIP \\
\hline & MYO5A & Het & NM_000259.3 & $\begin{array}{c}\text { c. } 3567+4 \mathrm{C}>\mathrm{T} \\
\text { p.? }\end{array}$ & unknown & rs186277072 & n.a. \\
\hline \multirow{2}{*}{ NA06110 } & \multirow{2}{*}{ SGSH } & Het & \multirow{2}{*}{ NM_000199.5 } & $\begin{array}{c}\text { c.734G }>\text { A } \\
\text { p.Arg245His }\end{array}$ & missense & rs104894635 & $\mathbf{P}$ \\
\hline & & Het & & $\begin{array}{c}\text { c.629G }>\text { A } \\
\text { p.Trp210Ter }\end{array}$ & nonsense & rs886041370 & $\mathrm{P} / \mathrm{LP}$ \\
\hline \multirow{3}{*}{ NA20379 } & \multirow{2}{*}{ PPT1 } & \multirow{2}{*}{ Het } & \multirow{2}{*}{ NM_000310.4 } & $\begin{array}{c}\text { c.364A>T } \\
\text { p.Arg122Trp }\end{array}$ & missense & rs137852695 & $\mathbf{P}$ \\
\hline & & & & $\begin{array}{c}\text { c.125G }>A \\
\text { p.Gly42Glu }\end{array}$ & missense & rs386833631 & $\mathbf{L P}$ \\
\hline & $G A A$ & Het & NM_001079804.3 & $\begin{array}{c}\text { c.525delT } \\
\text { p.Glu176ArgfsTer45 }\end{array}$ & frameshiftDeletion & rs386834235 & $\mathrm{P}$ \\
\hline \multirow{4}{*}{ NA03124 } & GUSB & Het & NM_000181.4 & $\begin{array}{c}\text { c. } 454 \mathrm{G}>\mathrm{A} \\
\text { p.Asp152Asn }\end{array}$ & missense & rs149606212 & US \\
\hline & \multirow{2}{*}{ NPC1 } & \multirow{2}{*}{ Het } & \multirow{2}{*}{ NM_000271.5 } & $\begin{array}{c}\text { c.3182T }>C \\
\text { p.Ile1061Thr }\end{array}$ & missense & rs80358259 & $\mathbf{P}$ \\
\hline & & & & $\begin{array}{c}\text { c. } 1947+5 G>C \\
\text { p.? }\end{array}$ & unknown & rs770321568 & CIP \\
\hline & $A R S A$ & Het & NM_001085425.3 & $\begin{array}{c}\text { c.698_699insC } \\
\text { p.Gln234SerfsTer41 }\end{array}$ & frameshiftInsertion & - & n.a. \\
\hline \multirow{3}{*}{ NA03111 } & \multirow{2}{*}{$L I P A$} & \multirow{2}{*}{ Het } & \multirow{2}{*}{ NM_001127605.3 } & $\begin{array}{c}\text { c.967_968delAG } \\
\text { p.Ser323LeufsTer44 }\end{array}$ & frameshiftDeletion & rs917089035 & n.a. \\
\hline & & & & $\begin{array}{l}\text { c. } 894 G>A \\
\text { p.Gln } 298=\end{array}$ & synonymous & rs116928232 & P/LP \\
\hline & GALNS & Het & NM_000512.5 & $\begin{array}{c}\text { c.499T>G } \\
\text { p.Phe167Val }\end{array}$ & missense & rs148565559 & US \\
\hline NA02057 & $A G A$ & Het & NM_000027.4 & $\begin{array}{c}\text { c.488G>C } \\
\text { p.Cys163Ser }\end{array}$ & missense & rs121964904 & $\mathbf{P}$ \\
\hline
\end{tabular}


Table 2. Cont

\begin{tabular}{|c|c|c|c|c|c|c|c|}
\hline $\begin{array}{l}\text { ID Coriell } \\
\text { Sample }\end{array}$ & Genes & Zigosity & Transcript & $\begin{array}{l}\text { Coding Amino Acid } \\
\text { Change }\end{array}$ & Variant Effect & dbSNP & ClinVar \\
\hline \multirow{4}{*}{ NA00879 } & BLOC1S6 & Het & NM_012388.4 & $\begin{array}{c}\text { c.225-2_225-1insT } \\
\text { p.? }\end{array}$ & unknown & - & n.a. \\
\hline & $S G S H$ & Het & NM_000199.5 & $\begin{array}{c}\text { c.1339G >A } \\
\text { p.Glu447Lys }\end{array}$ & missense & rs104894639 & $\mathbf{P} / \mathbf{L P}$ \\
\hline & $S G S H$ & \multicolumn{6}{|c|}{ Second Variant not detected c.746G>A (Arg245His (R245H)) } \\
\hline & CTSA & Het & NM_000308.4 & $\begin{array}{c}\text { c.263_264insG } \\
\text { p.Cys88TrpfsTer52 }\end{array}$ & frameshiftInsertion & - & n.a. \\
\hline NA01256 & IDUA & Het & NM_000203.5 & $\begin{array}{l}\text { c.590-7G>A } \\
\text { p.? }\end{array}$ & unknown & rs762411583 & $\mathrm{P}$ \\
\hline
\end{tabular}

$\mathrm{P}=$ pathogenic; $\mathrm{LP}=$ likely pathogenic; $\mathrm{US}=$ uncertain significance; $\mathrm{CIP}=$ conflicting interpretation of pathogenicity; n.a. = not available. True-positive variants are reported in bold, and new observed findings are reported in non-bold text.

From the run metrics results, all samples were uniformly covered at depths that exceed the minimum coverage required $(30 \times)$ for the accurate calling of variants. Coverage analysis shows that $1225 / 1241$ of the amplicons $(98.7 \%$ ) had a sufficient amplification efficiency (mean assigned reads per amplicon Log10 ranging from 1.5 to 3.8), while 16 amplicons were suboptimal (Figure 1 and Supplementary Table S2).

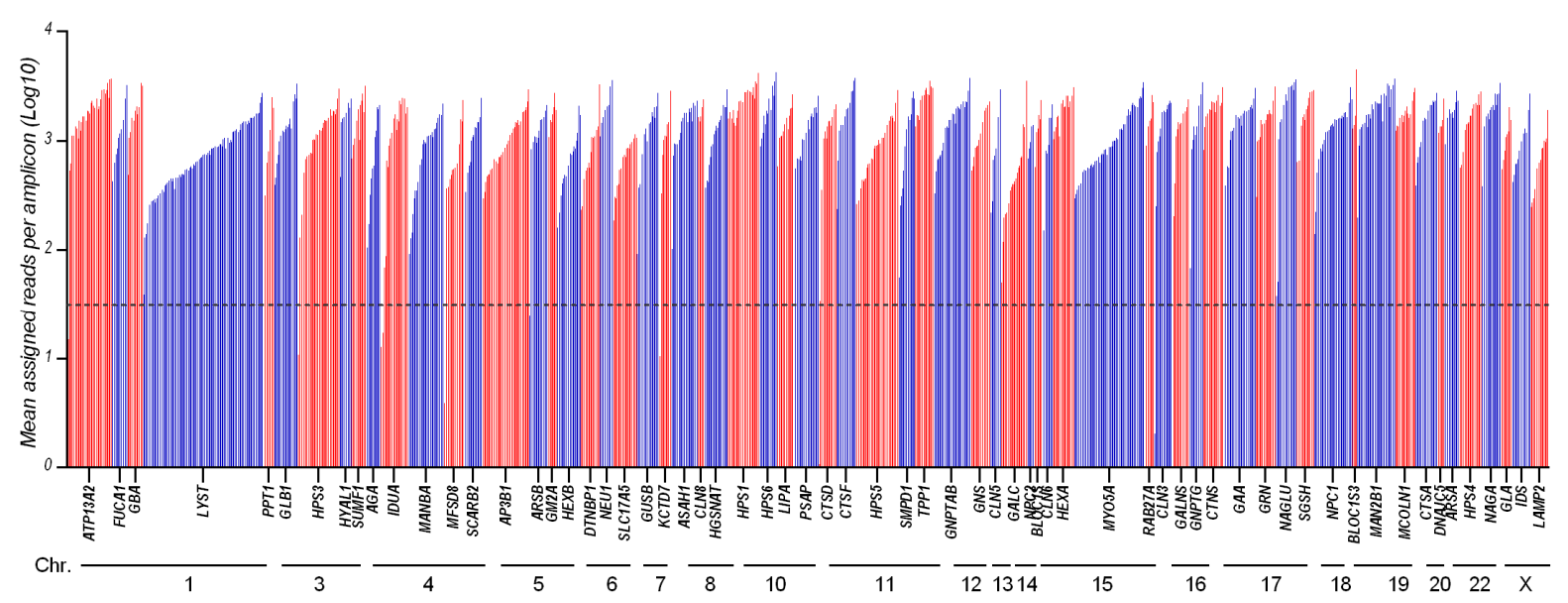

Figure 1. Amplicon coverage of the 65 targeted genes: 1241 amplicons distributed across 65 genes were amplified and sequenced with LSDs_panel. This chart shows the mean coverage of individual targeted amplicons across each gene for 26 analyzed samples. Amplicons with zero reads were arbitrarily represented as 0.

Filtering the pipeline on the TVC (Torrent Variant Caller, Thermo Fisher Scientific, Waltham, MA, USA) was based on a stepwise-adjusted strategy to highlight relevant variants (i.e., coverage min $30 \times$, homopolymer length $\leq 3$, $p$-value $<0.001$, ClinVar $\neq$ benign or likely benign, MAF $<0.001$ or none, frequency $30-60 \%$ for heterozygous variants and $>70 \%$ for homozygous variants, include intronic variants if the distance from exon is $<10 \mathrm{bp}$, SIFT score $<0.05$ or none, PolyPhen score $>0.85$ or none, and variants effect $\neq$ synonymous unless they are pathogenic/likely pathogenic/uncertain significance or with conflicting interpretation of pathogenicity). A comparison with the previously known variants reported in Coriell biobank was performed by post-filtering analysis. True-positive (TP), true-negative (TN), false-positive (FP), and false-negative (FN) variant calls were defined by considering the available data from a single causative gene in the Coriell repository (see Section 2).

The overall accuracy of the panel was $98.4 \%$, analytical sensitivity was $95.2 \%$, while specificity was $97.6 \%$. There were 40 correctly called true-positive variants, 83 true-negative reference calls, and 2 false-negative (missed) calls when comparing our results with the 
expected variants (Table 2). The MCC was 0.964 (MCC $=+1$ describes a perfect prediction, $=0$ means unable to return any valid information, and $=-1$ describes complete inconsistency between prediction and observation).

\subsection{Control Samples Analysis}

The majority of detected pathogenic mutations and polymorphisms are consistent with the data reported in the Coriell biobank. Interestingly, some additional observations in single causative genes emerged that are worthy to be mentioned in order to update data in the repository, as we describe below.

The NA06110 sample, acquired from Coriell biobank, derives from a female donor subject described as a compound heterozygote, with one allele carrying a G>A transition in the SGSH gene causing the Arg245His (R245H) aminoacidic variation and "no changes detected in the other allele". The LSDs_panel was able to successfully detect the $\mathrm{R} 245 \mathrm{H}$ change, identifying a second heterozygous mutation (i.e., the c.629G $>\mathrm{A}$, causing the nonsense aminoacidic change-p.Trp210Ter) reported as pathogenic/likely pathogenic in ClinVar (Table 2). Thus, in addition to confirming the previously detected variant, our analysis indicated the presence of another, extending the genotypic portrait of the sample.

An additional observation is with regard to the NA02057 DNA sample, which carries a pathogenic homozygous G-to- $C$ transversion in the $A G A$ gene, resulting in a substitution of serine for cysteine at codon 163 (Cys163Ser (C163S)). The Coriell biobank reports also a heterozygous G-to-A transition (Arg161Gln (R161Q)) in the same gene, which was detected by the LSDs_panel, but classified as benign in ClinVar.

The two false negative variants were detected in the NA00879 and NA01256 samples (Table 2). The first (c.746G>A (Arg245His [R245H])) was completely missed by sequencing, whereas the second (c.1293TGG>TAG (Trp402Ter [W402X])) was detected by the panel but excluded due to very low coverage (below the threshold of $30 \times$ ). We cannot rule out that missed genetic modifications are the result of high culture passages.

The LSDs_panel detected additional non-pathogenic variants in the analyzed samples (Table 2, in non-bold text) that may reduce enzymatic activity and may contribute to phenotypic manifestations. Given the variability of symptom manifestations as well as the phenotypic overlapping between genetically different disorders, the presence of additional secondary variants or genetic modifiers involved in lysosomal regulation and metabolism should be considered and may help to refine genotype-phenotype correlations.

\section{Discussion}

As outlined earlier, there are many factors hampering the diagnosis of LSDs, including the phenotypic and penetrance variability, the common signs and symptoms between certain disease groups, the genetic heterogeneity, and the difficulties of biochemical diagnostics. Developing a powerful diagnostic tool could mitigate the delayed diagnostic process for affected families, leading to better outcomes for current therapies and providing the basis for more appropriate genetic counseling. Many recent reports have emphasized the high clinical utility of NGS technologies and targeted gene panels in the diagnosis of suspected LSDs and their potential to reduce diagnostic delay [11-17].

Herein, we proposed a tNGS panel (LSDs_panel) based on AmpliSeq technologies to simultaneously screen the coding regions of 65 genes responsible for a heterogeneous group of LSDs and aimed at evaluating its clinical utility in suspected patients. By using a set $(n=26)$ of standard samples from Coriell Institute biobank (https: / / www.coriell.org/, accessed on 26 October 2021), we assessed the overall accuracy of the panel $(98.4 \%)$, the analytical sensitivity (95.2\%), and the specificity (97.6\%) of the NGS workflow. Known pathogenic mutations in the reference samples were identified with the correct homozygous/heterozygous state.

Several published papers have shown the possibility of carrying out successful NGS sequencing studies from DNA extracted from Guthrie card (DBS) fingerprints, thus taking advantage of the possibility of using the same non-invasive sampling from newborns for 
both biochemical and sequencing tests $[18,19]$. Preliminary experiments in our lab starting from DBS-isolated DNA and sequenced with the LSD panel showed adequate amplicon coverage, revealing the feasibility of the NGS approach when starting from dried samples.

A second-tier application of the comprehensive LSDs_panel may be in the field of modifier genes, complex disorders, and polygenic inheritance $[15,20,21]$. It is well known that patients who share the same mutations may have a different phenotypic spectrum. Thus, the effect of the primary molecular defects may be modified by the presence of additional cumulative mutations located in other genes that encode proteins involved in lysosomal pathways (Table 2). The possibility of detecting variants with uncertain significance and/or secondary findings should be, however, carefully considered in reporting the results, clearing the (probable) non-causality role of the mutation. The decision to report such mutations should always be in accordance with informed consent signed by patients.

A strong limitation of the panel is the poor ability to detect complex rearrangements and recombined genomic regions, which may all require other techniques for elucidation. $\mathrm{CNVs}$, including both deletions and amplifications, may be visualized starting from NGS data by manually checking the coverage of the suspected gene: the degree of coverage of the examined region with respect to the same region in other samples of the same run could suggest the presence of a CNV in heterozygous or homozygous state. However, in both cases, different molecular techniques should be used to confirm the suspected alterations as well as to exclude potential allelic dropout events.

Taken together, we demonstrated here that an NGS-based approach for the detection of LSDs may be a valuable adjunct test along with the well-established biochemical assays. Indeed, while enzyme analysis is still the gold standard for many LSDs (characterized by enzymatic deficiency), it may not accurately identify all obligate carriers and cannot be applied to disorders caused by alterations in transport or transmembrane (non-catalytic) proteins. That a broader spectrum of diseases can be monitored in one single test significantly shortens the analysis time for complex phenotypes or when a biochemical test cannot be offered. Finally, genotype-phenotype correlations may be carefully analyzed since they may be discordant, and clinicians should be cautious when counseling families regarding prognosis.

\section{Conclusions}

NGS technology is currently offering the opportunity to improve the LSD diagnostic workflow, given its low cost, semi-automated pipeline, short processing time, and ability to simultaneously detect multiple nucleotide variants on several genes. A broader adoption of targeted NGS-based tests, such as the assessment described here, should be taken into consideration to optimize clinical management of LSDs characterized by high levels of clinical and biochemical heterogeneity.

The use of targeted NGS may represent a real and valuable strategy for providing timely and correct diagnoses, for detecting carriership status, and for ensuring genetic counseling for family planning. Moreover, molecular profiling and genomic sequencing information may prompt the design of novel therapeutic drugs targeting specific mutations, thus opening the possibility for personalized medicine. Efforts in this sense may prompt patient-oriented outcomes, may improve the quality of life of patients and their families, and may reduce both direct and indirect costs (e.g., caregivers' services) to national health services and families.

Supplementary Materials: The following are available online at https:/ /www.mdpi.com/article/ 10.3390/genes12111750/s1, Table S1: Design (bed file) of the LSD panel; Table S2: Mean reads per amplicon.

Author Contributions: Conceptualization, V.L.C. and S.C.; data curation, V.L.C.; formal analysis, V.L.C.; funding acquisition, S.C.; investigation, V.L.C.; methodology, V.L.C.; resources, S.C.; supervision, S.C.; writing—original draft, V.L.C.; writing—review and editing, V.L.C. and S.C. All authors have read and agreed to the published version of the manuscript. 
Funding: This work was supported by the joint project between IRIB-CNR and SANOFI “Early diagnosis of some lysosomal diseases: analysis of the clinical utility and diagnostic validity of genomic techniques for their molecular diagnosis. Assessments of the implications of the inclusion of lysosomal diseases in the context of a national neonatal screening program" (project n. 2018/9848).

Institutional Review Board Statement: Not applicable.

Informed Consent Statement: Not applicable.

Data Availability Statement: Details of the reference samples selected for the present validation can be found at https:/ / www.coriell.org/ (accessed on 26 October 2021).

Acknowledgments: The authors gratefully acknowledge Cristina Calì, Alfia Corsino, Maria Patrizia D'Angelo, and Francesco Marino for administrative and technical support.

Conflicts of Interest: The authors declare no conflict of interest. The funders had no role in the design of the study, in writing the manuscript, or in the decision to publish the results.

\section{References}

1. Platt, F.M. Emptying the stores: Lysosomal diseases and therapeutic strategies. Nat. Rev. Drug Discov. 2018, 17, 133-150. [CrossRef] [PubMed]

2. Platt, F.M.; d'Azzo, A.; Davidson, B.L.; Neufeld, E.F.; Tifft, C.J. Lysosomal storage diseases. Nat. Rev. Dis. Primers 2018, 4, 27. [CrossRef]

3. Ferreira, C.R.; Gahl, W.A. Lysosomal storage diseases. Transl. Sci. Rare Dis. 2017, 2, 1-71. [CrossRef]

4. La Cognata, V.; Guarnaccia, M.; Polizzi, A.; Ruggieri, M.; Cavallaro, S. Highlights on Genomics Applications for Lysosomal Storage Diseases. Cells 2020, 9, 1902. [CrossRef]

5. Kingma, S.D.; Bodamer, O.A.; Wijburg, F.A. Epidemiology and diagnosis of lysosomal storage disorders; challenges of screening Best Pract. Res. Clin. Endocrinol. Metab. 2015, 29, 145-157. [CrossRef]

6. Pinto, E.V.F.; Rojas Malaga, D.; Kubaski, F.; Fischinger Moura de Souza, C.; de Oliveira Poswar, F.; Baldo, G.; Giugliani, R. Precision Medicine for Lysosomal Disorders. Biomolecules 2020, 10, 1110. [CrossRef] [PubMed]

7. Mokhtariye, A.; Hagh-Nazari, L.; Varasteh, A.R.; Keyfi, F. Diagnostic methods for Lysosomal Storage Disease. Rep. Biochem. Mol. Biol. 2019, 7, 119-128.

8. Waggoner, D.J.; Tan, C.A. Expanding newborn screening for lysosomal disorders: Opportunities and challenges. Dev. Disabil. Res. Rev. 2011, 17, 9-14. [CrossRef]

9. Komlosi, K.; Sólyom, A.; Beck, M. The Role of Next-Generation Sequencing in the Diagnosis of Lysosomal Storage Disorders. J. Inborn Errors Metab. Screen. 2016, 4, 4. [CrossRef]

10. Encarnação, M.; Coutinho, M.F.; Silva, L.; Ribeiro, D.; Ouesleti, S.; Campos, T.; Santos, H.; Martins, E.; Cardoso, M.T.; Vilarinho, L.; et al. Assessing Lysosomal Disorders in the NGS Era: Identification of Novel Rare Variants. Int. J. Mol. Sci. 2020, $21,6355$. [CrossRef]

11. Fernandez-Marmiesse, A.; Morey, M.; Pineda, M.; Eiris, J.; Couce, M.L.; Castro-Gago, M.; Fraga, J.M.; Lacerda, L.; Gouveia, S.; Perez-Poyato, M.S.; et al. Assessment of a targeted resequencing assay as a support tool in the diagnosis of lysosomal storage disorders. Orphanet J. Rare Dis. 2014, 9, 59. [CrossRef]

12. Zanetti, A.; D’Avanzo, F.; Bertoldi, L.; Zampieri, G.; Feltrin, E.; De Pascale, F.; Rampazzo, A.; Forzan, M.; Valle, G.; Tomanin, R. Setup and Validation of a Targeted Next-Generation Sequencing Approach for the Diagnosis of Lysosomal Storage Disorders. J. Mol. Diagn. 2020, 22, 488-502. [CrossRef] [PubMed]

13. Gheldof, A.; Seneca, S.; Stouffs, K.; Lissens, W.; Jansen, A.; Laeremans, H.; Verloo, P.; Schoonjans, A.S.; Meuwissen, M.; Barca, D.; et al. Clinical implementation of gene panel testing for lysosomal storage diseases. Mol. Genet. Genom. Med. 2019, 7, e00527. [CrossRef]

14. Levesque, S.; Auray-Blais, C.; Gravel, E.; Boutin, M.; Dempsey-Nunez, L.; Jacques, P.E.; Chenier, S.; Larue, S.; Rioux, M.F.; Al-Hertani, W.; et al. Diagnosis of late-onset Pompe disease and other muscle disorders by next-generation sequencing. Orphanet J. Rare Dis. 2016, 11, 8. [CrossRef]

15. Di Fruscio, G.; Schulz, A.; De Cegli, R.; Savarese, M.; Mutarelli, M.; Parenti, G.; Banfi, S.; Braulke, T.; Nigro, V.; Ballabio, A. Lysoplex: An efficient toolkit to detect DNA sequence variations in the autophagy-lysosomal pathway. Autophagy 2015, 11, 928-938. [CrossRef] [PubMed]

16. Malaga, D.R.; Brusius-Facchin, A.C.; Siebert, M.; Pasqualim, G.; Saraiva-Pereira, M.L.; Souza, C.F.M.; Schwartz, I.V.D.; Matte, U.; Giugliani, R. Sensitivity, advantages, limitations, and clinical utility of targeted next-generation sequencing panels for the diagnosis of selected lysosomal storage disorders. Genet. Mol. Biol. 2019, 42, 197-206. [CrossRef] [PubMed]

17. La Cognata, V.; Guarnaccia, M.; Morello, G.; Ruggieri, M.; Polizzi, A.; Cavallaro, S. Design and Validation of a Custom NGS Panel Targeting a Set of Lysosomal Storage Diseases Candidate for NBS Applications. Int. J. Mol. Sci. 2021, 22, 64. [CrossRef] [PubMed]

18. Hendrix, M.M.; Cuthbert, C.D.; Cordovado, S.K. Assessing the Performance of Dried-Blood-Spot DNA Extraction Methods in Next Generation Sequencing. Int. J. Neonatal Screen. 2020, 6, 36. [CrossRef] 
19. Boemer, F.; Fasquelle, C.; d'Otreppe, S.; Josse, C.; Dideberg, V.; Segers, K.; Guissard, V.; Capraro, V.; Debray, F.G.; Bours, V. A next-generation newborn screening pilot study: NGS on dried blood spots detects causal mutations in patients with inherited metabolic diseases. Sci. Rep. 2017, 7, 17641. [CrossRef]

20. Davidson, B.A.; Hassan, S.; Garcia, E.J.; Tayebi, N.; Sidransky, E. Exploring genetic modifiers of Gaucher disease: The next horizon. Hum. Mutat. 2018, 39, 1739-1751. [CrossRef]

21. Parenti, G.; Medina, D.L.; Ballabio, A. The rapidly evolving view of lysosomal storage diseases. EMBO Mol. Med. 2021,13 , e12836. [CrossRef] [PubMed] 